Rincón, C., Molina, F. y Narváez, J. (2020), Perspectiva de la Alianza Cooperativa Internacional sobre la aplicación de las NIIF en las empresas cooperativas. Contaduría Universidad de Antioquia, 76, 35-53.

Doi:https://doi.org/10.17533/udea.rc.n76a03

\title{
Perspectiva de la Alianza Cooperativa Internacional sobre la aplicación de las NIIF en las empresas cooperativas
}

\author{
Carlos Augusto Rincón Soto \\ carlos.augusto.rincon@correounivalle.edu.co \\ Universidad del Valle \\ orcid: 0000-0003-4710-5558
}

Francisco Ricardo Molina Mora

francisco.molina@correounivalle.edu.co Universidad del Valle

Orcid: 0000-0003-1998-3492

Julián Andrés Narváez Grisales

janarvaez@usbcali.edu.co

Universidad de San Buenaventura

orcid: 0000-0002-5817-7699 
Perspectiva de la Alianza Cooperativa Internacional sobre la aplicación de las NIIF en las empresas cooperativas

Resumen: El cooperativismo se ha distinguido por su discordancia ante los fundamentos económicos del capitalismo; por ende, ha configurado sus propios principios, formas de gobierno y representación económica. No obstante, los organismos de emisión de normas contables han expandido sus estándares de manera homogénea hacia todos los sectores, incluyendo las organizaciones sociales y solidarias. Este estudio pretende develar la posición de la Alianza Cooperativa Internacional por medio de los métodos de análisis de contenido y análisis del discurso en relación al acogimiento de las normas internacionales de información financiera por parte de las entidades cooperativas.

Palabras clave: Contabilidad cooperativa, regulación contable, Normas Internacionales de Información Financiera (NIIF), Alianza Cooperativa Internacional (ACI), International Accounting Standards Board (IASB).

Perspective of the International Cooperative Alliance on the application of IFRS in cooperative companies

Abstract: Cooperativism has been characterized by its discord with respect to the economic foundation of capitalism, hence it has framed its own principles, ways of governance and economic representation. However, regulatory bodies issuing accounting regulations have expanded their standards homogeneously to all sectors, including social and solidarity organizations. This study seeks to unveil the position of the International Cooperative Alliance through content and discourse analysis methods regarding compliance with international financial reporting standards by cooperative entities.

Keywords: Cooperative accounting, accounting regulations, International Financial Reporting Standards (IFRS), International Cooperative Alliance (ICA), International Accounting Standards Board (IASB).

\section{Perspectiva da "Associação Cooperativa Internacional” sobre a aplicação das NIIF nas empresas cooperativas}

Resumo: $O$ cooperativismo tem se caracterizado por sua discordância com os fundamentos econômicos do capitalismo; por isso, tem configurado seus próprios princípios, formas de governo e representação econômica. Mesmo assim, os organismos de emissão de normas contábeis têm expandido seus padrões de maneira homogênea a todos os setores, incluindo as organizações sociais e solidárias. Este estudo pretende revelar o posicionamento da "Associação Cooperativa Internacional" por meio dos métodos da análise de conteúdo e análise do discurso em relação ao acolhimento das Normas Internacionais de Informação Financeira às entidades cooperativas.

Palavras chave: Contabilidade Cooperativa, Regulação contábil, Normas Internacionais de Informação Financeira (NIIF), Associação Cooperativa Internacional (ACI), International Accounting Standards Board (IASB).

Perspective de l'Alliance Coopérative International sur l'application des IFRS dans les entreprises coopératives

Résumé: le coopérativisme se dintingue par sa discordance face aux bases économiques du capitalisme. Par conséquent, il a structuré ses propres principes, formes de gouvernement, ainsi que sa représentation économique. Néanmoins, les organismes d'émission des normes comptables ont élargi leurs standards de façon homogène vers tous les secteurs, organisations sociales et solidaires incluses. Cette étude vise à dévoiler la position de l'Alliance Coopérative Internationale au moyen des méthodes d'analyse du contenu et d'anaylse du discours liéés à l'acceptation des normes internationales de l'information financière aux entités coopératives.

Mots clés: comptabilité coopérative, régulation comptable, Normes internationales d'Information Financière (IFRS), Alliance Coopérative Internationale (ACI), International Accounting Standards Board (IASB). 


\title{
Perspectiva de la Alianza Cooperativa Internacional sobre la aplicación de las NIIF en las empresas cooperativas
}

\author{
Carlos Augusto Rincón Soto, Francisco Ricardo Molina Mora, \\ Julián Andrés Narváez Grisales \\ https://doi.org/10.17533/udea.rc.n76a03
}

Primera versión recibida en septiembre de 2019 - Versión aceptada en marzo de 2020.

\section{Introducción}

L os organismos de emisión de las normas de información financiera aprivilegian las posturas del mercado de capital, e inciden hacia un proceso homogeneizador de los marcos normativos contables a todos los sectores económicos, incluyendo las empresas del sector cooperativo.

Actualmente, las cooperativas están incluidas en las Normas Internacionales de Información Financiera (NIIF), que describen la situación financiera basada en las necesidades de los usuarios de las empresas con ánimo de lucro, en las que prima la rentabilidad como parte del discurso empresarial. En el marco conceptual, parte A de las normas internacionales, se establece que el usuario principal a quien va a satisfacer esta norma son los inversionistas, acreedores y prestamistas existentes o potenciales; la parte $\mathrm{B}$ menciona que el principal propósito de los estados financieros realizados bajo NIIF es mostrar solvencia, porque este objetivo está directamente alineado con las necesidades del usuario principal (IASB, 2010, pt. FC 1.34); esto justifica la jerarquía de la medición al valor razonable ante la primacía del mercado en las categorías contables (Gómez y Álvarez, 2013).

De la misma manera en el párrafo OB9 se establece que la gerencia no es un usuario directo interesado de las NIIF y el OB10 plantea que el regulador, o cualquier otro usuario, tampoco hará parte de los usuarios principales (IASB, 2010). Las NIIF se centran en proporcionar información económica para que los usuarios principales tomen decisiones financieras (IASB, 2010, pt. FC 1.24), competentes a su papel de suministradores de capital de riesgo, además de 
Rincón, C., Molina, F. y Narváez, J. Perspectiva de la Alianza Cooperativa Internacional...

evaluar los resultados de la gerencia respecto a los resultados financieros (IASB, 2010, pt. FC 1.26).

Algunos expertos plantean que las NIIF no se adecúan a las necesidades del sector cooperativo, y por ello convendría plantear sus propios marcos regulativos contables (Gómez y Miranda, 2006; Robb, 2012). Por consiguiente, es de esperar que La Alianza Cooperativa Internacional (ACI), que reúne y representa a las organizaciones cooperativas en el mundo, debería tener una postura clara sobre el devenir de la representación contable de las cooperativas.

Sobre el dilema de la aplicación de las NIIF para el sector cooperativo, sería oportuno plantearse los siguientes interrogantes: ¿El inversionista de una empresa privada es igual al asociado de una empresa sin ánimo de lucro? ¿Los inversionistas y asociados tienen las mismas necesidades de información financiera y contable? Las respuestas a estas preguntas dependen de la postura institucional, considerando que para el IASB ${ }^{1}$ puede ser lo mismo inversionista que asociado, mientras su aporte sea residual; de lo contrario, es un prestatario. La posición de la comunidad cooperativa, sobre este dilema, podría clarificar la aceptación o crítica al uso de las NIIF. Por ello, esta investigación tiene como objetivo identificar cuál es la postura de la $\mathrm{ACI}$, con respecto a la aplicación de las NIIF para el sector cooperativo.

Para el desarrollo de este trabajo se toma como corpus distintas publicaciones de la $\mathrm{ACl}$, que tratan sobre las orientaciones que han suministrado en el tema de la implementación de las NIIF a las empresas cooperativas en distintos momentos. La metodología usada es de tipo cualitativa, fundamentándose en el análisis de contenido temático y análisis del discurso, herramientas con las que se rastreó los núcleos de sentido con respecto a la posición favorable, neutra o desfavorable de la $\mathrm{ACl}$ en relación con la aplicación de las NIIF en las cooperativas y de los cuales se puede inferir cuál es el argumento que determina su posición actual, partiendo de la premisa: "las palabras significan mucho más de lo que dicen" (Santander, 2011, p. 208).

\section{El modelo empresarial cooperativo}

Las empresas solidarias son un tipo especial de estructura de propiedad que como señalan Bruque Cámara, Hernández Ortiz, Moyano Fuentes y Sánchez (2002) se rigen por "principios de gestión y control democráticos" (p. 132). Su principal interés consiste en crear una organización que trabaje en necesidades socio-económicas comunes, las cuales a su vez se reconocen como sociedades de economía solidaria (Palacios, Cardona A. y Sánchez, 2012).

1 El IASB (International Accounting Standards Board) comité de normas internacionales de contabilidad de la fundación IFRS, encargada de emitir las normas internacionales de información financiera. Tomado de: https://www.nicniif.org/home/iasb/que-es-el-iasb.html 
Para Agreda Moreno (2012), estas empresas "buscan la democratización del capital y hacer posible la apropiación de los medios de producción y el producto del trabajo" (p. 24); es decir, trabajar por el bienestar del hombre y no por el egoísmo del capital, lo que Lasserre (2008) llamaría: "la continua construcción de un hombre virtuoso".

Las entidades solidarias son instituciones empresariales sin ánimo de lucro que trabajan bajo un discurso de asignación de recursos de manera sustentable, equitativa e igualitaria, basada en la labor cooperativa, democrática y social de los asociados. Como ejemplo de estas entidades se encuentran las cooperativas, empresas asociativas de trabajo, los fondos de empleados, las asociaciones mutuales, entre otras (Gaitán Sánchez, 2014), cuyos objetivos son distintos a las organizaciones con ánimo de lucro. En las empresas de capital privado la asignación de recursos y la toma de decisiones se basan en el aporte de capital y la meta final es la rentabilidad; por el contrario, en las empresas solidarias cada asociado participa en las tomas de decisiones, sin importar el porcentaje de capital y la asignación de recursos se realiza de manera equitativa: su fin primordial es el bienestar de los asociados.

La ACI define las cooperativas como "una asociación autónoma de personas que se han unido voluntariamente para hacer frente a sus necesidades y aspiraciones económicas, sociales y culturales comunes por medio de una empresa de propiedad conjunta y democráticamente controlada" (Cooperativa de las Américas, s.f.) En este sentido la ACI propone también unos principios fundamentales de la organización cooperativa, los cuales consisten en: membresía abierta y voluntaria, control democrático de los miembros, participación económica de los miembros, autonomía e independencia, educación, formación e información, cooperación entre cooperativas, compromiso con la comunidad.

La economía solidaria representa una posibilidad para superar el desempleo y la marginalidad, proponiendo economías sostenibles y sustentables e incentivando un tipo de organización opuesta al modelo capitalista dominante; se enfoca en el trabajo solidario y colectivo construyendo un proceso en el que se obtengan beneficios comunes. Visto de esta forma soluciona problemas y fallas sociales, económicas y financieras existentes en el modelo dominante que el capitalismo reconoce, pero no tiene las herramientas para resolver (Dussel, 2013; Pérez-Pascual, 2015).

En el lanzamiento del año internacional de las cooperativas, la presidente de la ACI, Pauline Green (2011), mencionó tres aspectos claves que se deben trasmitir entre las organizaciones cooperativistas:

1. Las cooperativas, propiedad de sus socios, son un modelo empresarial serio y de peso. Por lo tanto, las cooperativas piden que el marco legal y 
Rincón, C., Molina, F. y Narváez, J. Perspectiva de la Alianza Cooperativa Internacional...

financiero, específico y único de las cooperativas, sea plenamente aceptado y reconocido en las políticas públicas y en la normativa.

2. Las cooperativas, propiedad de sus miembros, son empresas guiadas por valores. Nuestros valores son parte integral de nuestro modelo empresarial, y no simplemente una herramienta de marketing, bajo la forma del informe anual de responsabilidad social corporativa. Esos valores definen nuestra identidad y nuestra marca y son parte de nuestro ADN. Las cooperativas piden entonces, que su modelo empresarial sea promovido del mismo modo que el de las empresas tradicionales.

3. Nuestro modelo de gobernabilidad es liderado por las personas, en una época en que las personas, sobre todo los jóvenes, a quienes la recesión está golpeando tan duramente, son reacios a los modelos económicos y políticos que dominan sus vidas. En momentos en los que están buscando hacerse oír en África del Norte, en Oriente Medio, en Wall Street y por todo el mundo, cuando están en la búsqueda de un impacto, la cooperativa no es sólo un modelo eficaz de gestión, sino un modelo en el que pueden creer. Las cooperativas piden que haya una mayor diversificación de la economía global, para asegurar igualdad de condiciones a su modelo empresarial de propiedad de los socios.

\section{Contabilidad cooperativa}

Es necesario definir qué es la contabilidad. Autores como Suárez Llanos y Manso Olivan (1998) la definen como una representación colectiva de la realidad que se establece a través de un lenguaje específico, porque "toda representación es un lenguaje" (p. 116) que transmite información y contenidos codificados; en este sentido, la contabilidad presenta, a través de informes, el valor comercial, productivo y social de una empresa, basándose en la necesidad de los usuarios que buscan en ella un respaldo para tomar decisiones. En este campo, el concepto de lenguaje resulta complejo; sin embargo, se trata de introducir a la contabilidad en un sistema de signos, con una estructura ordenada y significados que describen la situación financiera y no financiera de las organizaciones (López Pérez y Rodríguez Ariza, 2003; Rincón Soto, 2016). Ijiri (1975) manifiesta que la investigación de la contabilidad como lenguaje lograría conducir a una mayor claridad conceptual.

Los informes contables son el resultado de un proceso de recolección, manipulación y extracción de datos que se convierten en información. La regulación NIIF estandariza las políticas de reconocimiento, medición, revelación y presentación de las categorías contables asentadas en la mirada de la empresa con ánimo de lucro, basada en la propiedad privada. Las NIIF en la mayoría de los países regula la contabilidad cooperativa con la misma estandarización de políticas; solo tiene una cláusula especial para este sector 
en la sección 22.6 de las NIIF para PYME, o en la NIC 32 y la CINIIF 2 de las NIIF plenas, en la que dictamina como política contable, que el aporte de los asociados únicamente es patrimonio cuando este no se puede retirar, de lo contrario es un pasivo. Esta política se ha puesto en disputa debido al impacto que podría producir en las cooperativas.

Algunos autores están de acuerdo con la propuesta de las NIIF, porque se introduce a las cooperativas en la lógica del juego financiero y competitivo, pues buscan la estabilidad financiera a través del fortalecimiento del capital (Rendón Álvarez, Montaño Orozco y Gaitán León, 2013); otras posturas proponen ajustes a las políticas de revelación y presentación de las NIIF, para integrar particularidades como los fondos, reservas y cambio de algunas palabras como la de resultados por excedentes (Zubiaurre Artola, 2004). Por el contrario, hay investigadores que reprueban la postura de las NIIF, al determinar que simbólicamente los aportes en el pasivo no muestran la distinción del modelo cooperativo (Gómez y Miranda, 2006).

La propuesta de plantear los aportes recuperables de los asociados como pasivos, establece políticas para limitar las devoluciones, llevando a que las cooperativas incrementen medidas que fortalezcan el capital propio de la organización (Bastidas Vialcanet y Amat Salas, 2014), sacrificando otros proyectos, situación que podría impactar en el posible retiro y en la disminución de captura de nuevos asociados.

Para los autores de la contabilidad social, la información financiera es insuficiente para las necesidades de las organizaciones sin ánimo de lucro (Fernández-Lorenzo y Geba, 2005; Hernández Rodríguez y Escobar Castillo, 2017), porque la información de la gestión social se relaciona directamente con el objetivo cooperativo (Mirabal-González, Alfonso-Alemán y BarriosHernández, 2011) y hace parte de las necesidades de información de los asociados para que puedan tomar decisiones financieras y económicas de la organización. Mirabal (2014) analiza este tipo de información como otro subsistema del sistema contable.

Algunas posturas (Beaubien, 2011; Robb, 2012) rechazan la adecuación de la contabilidad cooperativa al modelo de contabilidad financiera bajo el razonamiento de discursos distintos al del sector con ánimo de lucro. "La relación entre los efectos sociales del poder corporativo y el papel de las organizaciones sin fines de lucro requiere más elaboración" (Valentinov, Hielscher y Pies, 2015, p. 5). Robb (2012) también expone que la contabilidad para el sector cooperativo es distinta al sector privado, porque se acerca más a una contabilidad administrativa de gestión, que a una contabilidad financiera.

Las NIIF en su marco conceptual plantean los siguientes principios contables: comprensibilidad, relevancia, fiabilidad y comparabilidad (IASB, 2010) como atributos indispensables que hacen útil la información para los 
Rincón, C., Molina, F. y Narváez, J. Perspectiva de la Alianza Cooperativa Internacional...

usuarios inversionistas, proveedores y acreedores existentes y potenciales. Sin embargo, las normas contables NIC/NIIF no comprenden la identidad del sector solidario y por lo tanto no ofrecen la información relevante para gestionar las metas y principios del cooperativismo. Entonces, ¿qué fiabilidad puede tener la información NIIF para el sector solidario, si no es comprensible, relevante y comparable, si como consecuencia hay mayor irregularidad de la información entre la contabilidad cooperativa y sus fines?

El capitalismo ve al asociado de la cooperativa como un cliente común, por lo cual supone que su aporte es un pasivo, una deuda; en este sentido el calificativo de aporte de los asociados bajo las categorías contables de las NIIF solo permite una posición financiera: pasivo o patrimonio. Para el capitalismo y sus instituciones representativas, siempre ha sido incomprensible el discurso solidario, entonces, ¿cómo el cooperativismo espera que el capitalismo le construya estándares de información con los cuales leer y controlar su tipología organizacional?

La contabilidad cooperativa se enfrenta hoy a un desafío debido a la imposición de estándares internacionales de información financiera desarrollados para y por compañías propiedad de inversores. Dichas normas no son "sectoriales neutrales" y deben ser resistidas para que las juntas cooperativas puedan informar de manera significativa. (Robb, 2012, p. 25)

\section{Tratamiento de estudio}

El objetivo del trabajo es observar cuál ha sido la posición de la ACI con respecto a la aplicación de las NIIF en las empresas del sector cooperativo, porque las NIIF representan instituciones que están basadas en el discurso capitalista, contradictorio en muchos aspectos con el discurso cooperativista.

Las organizaciones cooperativas han trabajado por posicionarse como organizaciones con un sentido distinto a las empresas con ánimo de lucro, por lo que situarse en un mismo plano de representación contable puede disponerlas como organizaciones semejantes en términos financieros, tributarios y hasta sociales. Por esto, el estudio de los distintos argumentos manifestados por la $\mathrm{ACI}$ es la base para analizar y deducir cuál será la orientación de las organizaciones cooperativas en la aplicación de las NIIF.

Las referencias bibliográficas tomadas para el análisis son publicaciones realizadas a través de plataformas web, las cuales evidencian las discusiones que se han dado en diferentes espacios como reuniones, talleres, congresos, publicaciones en revistas, convocados por la $\mathrm{ACl}$ o por instituciones que la representan, en el tema de la aplicación de las NIIF para el sector cooperativo.

Para el desarrollo de este estudio se tomaron las referencias que aparecen en el Cuadro 1. 
Cuadro 1: Referencias bibliográficas sobre ACl y la aplicación de la contabilidad NIIF.

\begin{tabular}{|c|c|c|c|}
\hline Doc & Fecha & Nombre & Link \\
\hline A1 & 21 Sep, 2005 & $\begin{array}{l}\text { Primera reunión del Grupo de } \\
\text { Normas Internacionales de } \\
\text { Contabilidad de la } \mathrm{ACl}\end{array}$ & $\begin{array}{l}\text { http://www.aciamericas.coop/Primera-reunion- } \\
\text { del-Grupo-de }\end{array}$ \\
\hline A2 & 30 Nov, 2005 & $\begin{array}{l}\text { Taller sobre Impacto de la NIC } 32 \\
\text { en el movimiento cooperativo }\end{array}$ & $\begin{array}{l}\text { http://www.aciamericas.coop/Taller-sobre- } \\
\text { Impacto-de-la-NIC-32?tema }=37 \& n b=65\end{array}$ \\
\hline A3 & $21 \mathrm{Nov}, 2005$ & $\begin{array}{l}\text { Seminario internacional sobre } \\
\text { impacto de las nuevas normas } \\
\text { internacionales de contabilidad en } \\
\text { las cooperativas, Buenos Aires }\end{array}$ & $\begin{array}{l}\text { http://www.aciamericas.coop/IMG/ } \\
\text { DeclaraciondeBsAs22-11-05.pdf }\end{array}$ \\
\hline A4 & $17 \mathrm{Jul}, 2006$ & $\begin{array}{l}\text { XIV Conferencia regional } \\
\text { competitividad y posicionamiento } \\
\text { cooperativo }\end{array}$ & http://www.aciamericas.coop/IMG/ConcluM2.pdf \\
\hline A5 & $17 \mathrm{Jul}, 2006$ & $\begin{array}{l}\text { Informe de parlamentarios de la } \\
\text { Alianza Cooperativa Internacional }\end{array}$ & $\begin{array}{l}\text { http://www.aciamericas.coop/IMG/declaracion_ } \\
\text { IV_encuentro_parlamentarios-2.pdf }\end{array}$ \\
\hline A6 & 30 May, 2008 & Correspondencia de ICA al IASB & $\begin{array}{l}\text { www.aciamericas.coop/.../ } \\
\text { ICApositionontheFASBpreliminaryvie }\end{array}$ \\
\hline A7 & Dic, 2008 & $\begin{array}{l}\text { Grupo de trabajo regional NIC-ACI } \\
\text { Américas }\end{array}$ & $\begin{array}{l}\text { http://www.aciamericas.coop/Documentacion- } \\
\text { del-Grupo-de-Trabajo }\end{array}$ \\
\hline A8 & 5 Ago, 2012 & $\begin{array}{l}\text { Conclusiones del XII Congreso } \\
\text { Nacional Cooperativo, Colombia }\end{array}$ & $\begin{array}{l}\text { http://www.aciamericas.coop/Conclusiones-del- } \\
\text { XII-Congreso }\end{array}$ \\
\hline B1 & Ago, 2013 & Confecoop Norte & $\begin{array}{l}\text { http://www.confecoopnorte.com.co/periodico/ } \\
\text { Periodico_Ed_35.pdf }\end{array}$ \\
\hline A9 & 2014 & $\begin{array}{l}\text { Propuesta para la coordinación } \\
\text { cooperativa internacional respecto } \\
\text { a las Normas Internacionales de } \\
\text { Contabilidad }\end{array}$ & $\begin{array}{l}\text { http://www.aciamericas.coop/lMG/pdf/ } \\
\text { PropuestaIASB_ES.pdf }\end{array}$ \\
\hline A10 & 2014 & $\begin{array}{l}\text { Declaración Cooperativa de } \\
\text { Cartagena } 2014\end{array}$ & http://www.aciamericas.coop/IMG/pdf/OAlpa.pdf \\
\hline A11 & 2014 & $\begin{array}{l}\text { Cooperativas de las Américas, } \\
\text { Región de la alianza cooperativa } \\
\text { internacional }\end{array}$ & $\begin{array}{l}\text { http://www.aciamericas.coop/lMG/pdf/ } \\
\text { informe_2014_de_actividades.pdf }\end{array}$ \\
\hline A12 & Dic, 2015 & $\begin{array}{l}\text { Revista de Integración Cooperativa } \\
\mathrm{N}^{\circ} 22 \text { Tema: La alianza contará con } \\
\text { una representación permanente en } \\
\text { el consejo consultivo que asesora } \\
\text { las NIIF }\end{array}$ & $\begin{array}{l}\text { https://issuu.com/jcse.info/docs/numero_22 } \\
\text { https://www.aciamericas.coop/La-Alianza- } \\
\text { contara-con-una-representacion-permanente- } \\
\text { en-el-Consejo-Consultivo }\end{array}$ \\
\hline B2 & 7 Ene, 2016 & $\begin{array}{l}\text { Excepción al tratamiento bajo NIIF } \\
\text { de la cartera de créditos y de los } \\
\text { aportes sociales en cooperativas }\end{array}$ & $\begin{array}{l}\text { http://confecoop.coop/comunicoop/2016/flash- } \\
\text { confecomunica-no-2019/ }\end{array}$ \\
\hline
\end{tabular}

Fuente: Elaboración propia.

En el Cuadro 1 los documentos aparecen clasificados cronológicamente para realizar un seguimiento ordenado de acuerdo con las fechas de publicación. Los documentos referenciados hacen parte de publicaciones entre los años 2005 y 
Rincón, C., Molina, F. y Narváez, J. Perspectiva de la Alianza Cooperativa Internacional...

2016, en los que se puede observar cuál ha sido la orientación de las posiciones argumentativas que ha presentado la $\mathrm{ACI}$ con respecto al tema de la aplicación de las NIIF en el sector cooperativo durante ese período. Las codificaciones por A son documentos de ACI y las codificaciones por B, son documentos publicados por la organización Confecoop que representa a la $\mathrm{ACI}$ en Colombia.

En su mayoría los informes destacan tácitamente lo concerniente al conflicto de posturas en relación con las aplicaciones de la NIIF en el sector cooperativo. A continuación se esboza una muestra de las frases o enunciados que permiten desarrollar el análisis. En el Cuadro 1 aparece el enlace a cada uno de los textos usados que respaldan esta investigación:

- 17 de julio de 2006, en el Informe de Parlamentarios de la ACI se destaca:

Lo establecido últimamente en la NIC 32 pretende incluir al capital cooperativo como pasivo y no como patrimonio neto. Esta norma produce un grave problema económico financiero a las cooperativas, cambiando sus índices de solvencia y endeudamiento, modificando el criterio de confidencialidad y haciendo que el aporte del asociado se convierta en un simple compromiso con terceros, violando el régimen de propiedad y el ejercicio de los plenos derechos políticos (elegir y ser elegido), una persona un voto.

- 8 de diciembre de 2008, Grupo de Trabajo Regional NIC-ACI Américas:

La NIC 32 se está analizando conjuntamente con las otras normas internacionales a partir del acuerdo para uniformar normas entre IASB (emisor de las NIC) y FASB (normas norteamericanas). Es por ello que a partir de este análisis permanente $\mathrm{ACI}$, a través de su grupo de trabajo, ha enviado notas tanto al IASB como al FASB para explicar la naturaleza específica de las cooperativas para que sea tratada como tal en las normas internacionales.

- $\quad$ Agosto de 2013, Confecoop Norte:

La adopción de las NIIF ofrece una oportunidad para mejorar la función financiera a través de una mayor consistencia en las políticas contables, obteniendo beneficios potenciales de mayor transparencia, incremento en la comparabilidad y mejora en la eficiencia.

La metodología utilizada fue análisis de contenido, usando frases temáticas de los documentos bajo frecuencia simple, donde se analizó la intensidad de los argumentos con respecto a la implementación de las NIIF en el sector cooperativo y la aplicación de la NIC 32 en la política de los aportes como pasivo y no como patrimonio; se evaluó cada pregunta a partir de tres posiciones: favorable, desfavorable y neutra. Luego, se develó el discurso no tácito que había en las posturas de los documentos.

Las preguntas y clasificaciones presentadas fueron: 
1. ¿El documento está de acuerdo con la contabilidad NIIF para la descripción de la información cooperativa?
a) Favorable
b) Desfavorable
c) Neutro

2. ¿El documento está de acuerdo con contabilizar el capital social igual a la NIC 32?
a) Favorable
b) Desfavorable
c) Neutro

\section{Resultados}

El estudio de los 12 documentos clasificados en "A" (Gráfico 1), arrojó 26 frases temáticas de las cuales 8 desaprobaban la aplicación de las NIIF (puntaje -1), 8 las aprobaban pero con cambios (puntaje 0), 9 consideraban favorable la aplicación de las NIIF a las empresas cooperativas (puntaje 1), y solo 1, en el primer documento, fue neutral a cualquier posición de las NIIF (no se integró en el Gráfico 1) y planteaba que se debía estudiar el impacto de la implementación para el sector cooperativo de manera urgente.

El Gráfico 1 representa los enunciados tenidos en cuenta para el estudio, y su calificativo en relación con la aprobación valorada con 1 o su desaprobación valorada con -1; en relación con la aplicación de las NIIF, además, cada punto en el Gráfico contempla el año en el que el organismo, que representa a las entidades sin ánimo de lucro, mencionó tal postura y se presenta de manera cronológica.

Gráfico 1: Nivel de favorabilidad cronológica de la ACI con respecto a las NIIF.

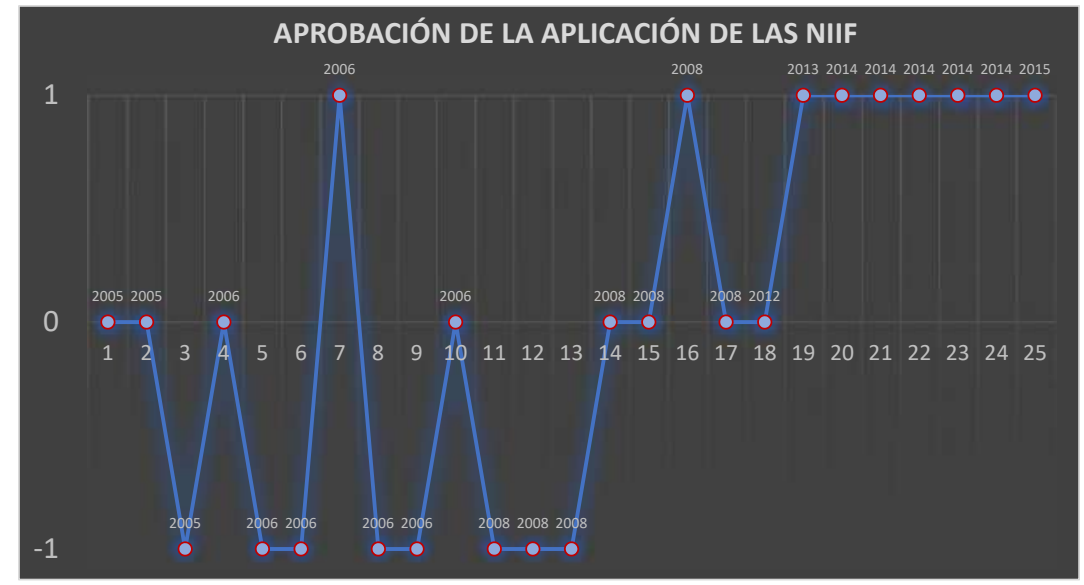

Fuente: Elaboración propia. 
Rincón, C., Molina, F. y Narváez, J. Perspectiva de la Alianza Cooperativa Internacional...

Las primeras discusiones dan a entender que el sector cooperativo se debe apartar de las NIIF, llevándolo a fundamentar sus propias normas contables, que reconozcan su identidad: "a las cooperativas se las trata como una organización de capital y es ahí donde, desde la $\mathrm{ACI}$, debemos hacer un trabajo a largo plazo y no solo actuar como lo hemos estado haciendo en los últimos años" (Cooperativas de las Américas, 2005). Analizar a las cooperativas de manera contable, igual que a las organizaciones con ánimo de lucro, aparece en los primeros documentos de la $\mathrm{ACI}$ como un despropósito:

...el organismo emisor de las Normas Contables Internacionales dirige sus acciones al objetivo de brindar información financiera para inversores de los mercados globales de capital, desconociendo las características esenciales de las cooperativas, razón por la cual no pueden ser adoptadas literalmente como normas contables para estas entidades. (Seminario Internacional sobre Impacto de las Nuevas Normas Internacionales de Contabilidad en las Cooperativas, 2005)

La no viabilidad de que se incluyan las cooperativas al marco contable NIIF, se expone en enunciados como: "resultan notoriamente extrañas a la particular naturaleza de las cooperativas" (XIV Conferencia Regional Competitividad y Posicionamiento Cooperativo, 2006, p. 2) o declaraciones como: "por ello no es admisible su inclusión como empresas de lucro, que define las normas internacionales de información financiera" (p. 3). Sin embargo, se puede observar dentro de este mismo documento expresiones que dejan ver otra intencionalidad: "resulta menester reclamar que esas normas armonicen con la Declaración Universal de Identidad Cooperativa" (p. 2); y en la última parte del documento: "el cual sugiere que el IASB haga partícipe a las entidades cooperativas -incorporando a sus representantes- en la elaboración de las normas contables" (p. 4); esto evidencia que desde un comienzo la posición política no era rechazar a las NIIF, sino la búsqueda de una posición dentro de la mesa de discusión.

Un año después, en la XIV Conferencia Regional de Competitividad y Posicionamiento Cooperativo (2006), las proposiciones de los resultados de las discusiones continúan siendo de rechazo a las NIIF; frases como: "el grave problema que se observa en las cooperativas es que estas normas están dirigidas a los mercados globales para todas las empresas de lucro, e incluso crean confusiones en sus normas respecto de las cooperativas con las mutuales" (p. 1), son expresiones que parecen irreconciliables entre dos posturas contradictorias: capitalismo y cooperativismo, que dejaban ver lo que parecía incompatible.

La metodología aplicada por la normativa está pensada para empresas internacionales con fines de rentabilidad de capital y no para cooperativas que se fusionan para cumplir mejor su objetivo social, lo que produce una gran distorsión e injusticia para ellas. (p. 2) 
O declaraciones como: "recomendamos no aplicar normas generales de contabilidad en los países, cuando estas normas internacionales son generadas principalmente para empresas que cotizan en bolsa de mercados globalizados" (p. 2). Estas frases muestran que la finalidad no era negociar con el IASB; pero, nuevamente se contradice, repitiendo la frase que se encontró en informes anteriores: "resulta menester reclamar que esas normas armonicen con la Declaración Universal de Identidad Cooperativa" (p. 2).

Hacia el año 2008, en la correspondencia de la ACI con IASB, se solicitó que tuvieran en cuenta las necesidades del sector cooperativo, permitiendo entrever así el deseo de negociar y ser partícipe en el proceso, solicitando a FASB (Financial Accounting Standards Board), entidad privada que labora en pro de mejorar los principios de contabilidad generalmente aceptados, "a considerar enfoques alternativos que puedan acomodar más fácilmente las diferentes estructuras de negocios y, sin embargo, permitir que FASB logre su objetivo de utilizar un enfoque uniforme para todo tipo de entidades" (Barberini, 2008, p. 4). También en esta correspondencia se reconoce la intención del IASB de apoyarlos al incluir la CINIIF 2, para solucionar, en algunos casos, la contabilización de los aportes como patrimonio, como si todo el problema de revelación de las cooperativas se debiera a la contabilización de los aportes sociales como pasivos.

Lo crítico es la posible sumisión de algunos de los principios cooperativos para poder cumplir el estándar contable homogeneizador. "La comunidad cooperativa internacional se unió e hizo compromisos significativos para cumplir con la CINIIF 2. De hecho, para muchas cooperativas, la CINIIF 2 forzó algunos cambios en sus leyes nacionales o regionales, estatutos y estructura de su organización” (Barberini, 2008, p. 6). Por último, en ese mismo documento, se reconoce la posición de aliado político: "Esperamos trabajar con FASB para garantizar que las normas internacionales de contabilidad tengan en cuenta las necesidades de la comunidad cooperativa internacional" (Barberini, 2008, p. 7).

A partir del año 2008 no se encontraron proposiciones negativas en las publicaciones de la $\mathrm{ACI}$ hacia la aplicación de las NIIF y el problema se centra en el tema de los aportes como patrimonio. Confecoop evalúa las NIIF de manera positiva: "La adopción de las NIIF ofrece una oportunidad para mejorar la función financiera a través de una mayor consistencia en las políticas contables obteniendo beneficios potenciales" (Anónimo, 2013, p. 10), teniendo en cuenta que básicamente el único cambio al que apela en todo el proceso hacia el sector cooperativo es la CINIIF 2, en tanto que este cambio obliga a afectar de algún modo unos de sus principios cooperativos: "en atención a lo que se ha venido manifestando en los diferentes seminarios, las entidades han venido incrementando su capital mínimo para el paso a las nuevas normas internacionales NIIF con el objetivo de seguir siendo viables" (p. 11). 
Rincón, C., Molina, F. y Narváez, J. Perspectiva de la Alianza Cooperativa Internacional...

Partiendo de este enunciado se evalúa la viabilidad de las cooperativas sobre su adecuación a las NIIF: lo que era antes negativo, ahora es positivo. Se ve claro que una de las metas de la participación de la ACI en el IASB, es descartar esta política contable hacia las cooperativas.

Después del año 2012 parece que los argumentos se posicionaron en que se debía implementar las NIIF y después de este año no aparecen otras expresiones ni documentos donde la $\mathrm{ACI}$ continúe generando controversia; esto puede ser porque a partir del año 2012 el IASB empieza a negociar un espacio en la mesa de discusión para la $\mathrm{ACI}$, cuestión que aparece concretada en los documentos del 2014.

En el año 2014 se consolida la alianza de la ACI con el IASB, y las proposiciones se vuelven más positivas hacia la implementación: "Las cooperativas han ganado terreno a nivel mundial en el acercamiento con los organismos que establecen las normas." (Propuesta para la Coordinación Cooperativa Internacional respecto a las Normas Internacionales de Contabilidad, 2014, p. 1); ahora esas normas pasan a ser efectivas para las cooperativas, aunque sean las mismas, pues siguen estando dirigidas al mercado bursátil, su principal objetivo son los usuarios inversionistas y el principal propósito de los estados financieros es mostrar solvencia.

El último proyecto de cambio del marco conceptual lo ratifica la fundación IFRS (2015). En ninguna parte se da lugar a las cooperativas dentro de un posible usuario principal. Pero los enunciados de la ACI continúan tomando partido sobre las NIIF: "en definitiva, la iniciativa respecto al futuro de las normas globales de contabilidad, está en nuestras manos" (Alpa, 2014, p. 37). Y en el 2015 el proyecto se abalanza hacia el cumplimiento de las NIIF y la participación permanente de la ACI: "necesitamos replicar estos esfuerzos a nivel internacional de la misma manera en que el IASB se posiciona en su papel de emisores de normas contables a nivel internacional. La ACI puede asumir el liderazgo como coordinador de este esfuerzo de una forma más significativa" (Cooperativas de las Américas, 2015).

En tanto la ACI tiene una participación constante en el proceso político de la implementación de normas NIIF de acuerdo a lo expresado en el boletín de las Cooperativas de las Américas:

...con el propósito de uniformizar la aplicación de las normas contables en todo el mundo, de manera que sean globalmente aceptadas, comprensibles y de alta calidad. Las NIIF permiten que la información de los estados financieros sea comparable y transparente, lo que ayuda a que los inversores y otros agentes que participan de los mercados de capitales de todo el mundo tomen sus decisiones. (Integración Cooperativa, 2015, p. 11)

Hubo un punto de inflexión donde las NIIF se volvieron positivas, pero las políticas normativas siguieron básicamente siendo las mismas. 
La pretensión política de la ACI de pertenecer al IASB, se expresa en varios documentos como una constante. Es una tendencia discursiva que no se esperaba hallar dentro del estudio; tanto así, que se volvieron a analizar los documentos para observar detenidamente esta intencionalidad, porque en el primer documento se menciona que se debe "asegurar el reconocimiento y la comprensión de la naturaleza especial de las empresas cooperativas por parte de la IASB... similar al reconocido por las Naciones" (Cooperativas de las Américas, 2005). Y a partir de los documentos del 2015, se concreta un discurso persuasivo sobre las ventajas de las NIIF, donde además se celebra el lugar otorgado por el IASB a la $\mathrm{ACl}$ en su mesa de discusión y se refiere como "un logro transcendental para el movimiento cooperativo mundial" (Integración Cooperativa, 2015, p. 11).

Con respecto al punto 2 sobre la aprobación de los aportes como pasivo, el dilema ha sido continuo y se mantiene un rechazo hacia la aplicación de esta política por parte de la $\mathrm{ACI}$ (Gráfico 2); es básicamente la única discusión que se mantiene; durante los años 2012 y 2013 hubo dos documentos de la $\mathrm{ACI}$ y de Confecoop que dejaban ver un tipo de adherencia hacia la aplicación de los aportes como pasivo y se proponían medidas internas que podían realizar las cooperativas para poder contabilizar los aportes como patrimonio en cumplimiento de la normatividad NIIF:

Determinar el efecto de las NIIF en la estructura financiera de las cooperativas, de manera que no se disminuya su patrimonio ante el eventual riesgo que los aportes sociales sean considerados un pasivo financiero. De ser así, trabajar en modificaciones a la ley que faciliten el fortalecimiento del capital institucional y dar plena claridad al manejo contable de los aportes, así como al procedimiento de cesión y devolución en caso de retiro de asociados. (Cooperativas de las Américas, 2012)

Gráfico 2: Cronología de aprobación de los aportes de los asociados como pasivo.

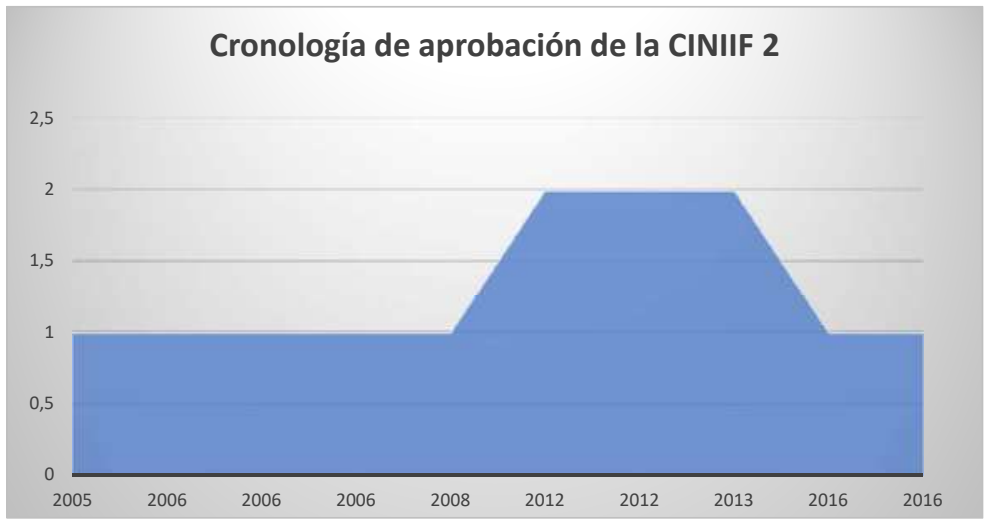

Fuente: Elaboración propia. 
Rincón, C., Molina, F. y Narváez, J. Perspectiva de la Alianza Cooperativa Internacional...

\section{Discusión y conclusiones}

El análisis de contenido cronológico de los documentos encontrados sobre la posición de la $\mathrm{ACI}$ en la aplicación de las normas internacionales de información financiera permite concluir una posición dócil por parte del ente representativo, con respecto a la postura de establecer su propio marco regulativo contable adecuado a las necesidades, intenciones e intereses del sector cooperativo. Esto en vista de que en los primeros documentos que se encontraron entre 2005 y 2008 , las proposiciones de la $\mathrm{ACI}$ fueron radicalmente negativas ante la implementación de las NIIF y algunas frases se advertían inamovibles (teniendo en cuenta que las NIIF se enfocan a las necesidades de los inversionistas, orientadas al mercado bursátil, de discurso netamente capitalista), y al final terminaron inclinándose a su aplicación.

Era de esperarse que en discursos tan discordantes, capitalismo y cooperativismo, colisionaran en el tema de su representación contable. Sin embargo, se percibe desde el primer documento de la $\mathrm{ACl}$, una intención tácita de cooperar con el IASB. El estudio de los documentos a través de su cronología, manifiesta esta intención al pasar de la discusión de rechazo de las NIIF a una aceptación y luego al elogio, como un grupo de políticas que representan las necesidades de información de las empresas cooperativas.

Este documento exterioriza la postura transitoria de oposición de la ACI frente a la imposición de las NIIF, porque en los inicios de la discusión prevalecía una actitud que negaba la posibilidad de una representación financiera basada en las entidades con ánimo de lucro, que no refiere a los mismos intereses de las entidades a las que representa la ACI. Esta postura es invertida, y a partir del año 2012, muestra una aceptación y condescendencia con las NIIF.

En la actualidad continúa siendo la política de los aportes como pasivo básicamente el único impedimento que tienen las normas ante la representación de las empresas cooperativas, al tiempo que se convirtió en la herramienta de batalla de la $\mathrm{ACI}$ en su representación ante el IASB.

El resultado de este trabajo servirá como precedente al momento de determinar cuáles serán las posibles pretensiones y controversias de la $\mathrm{ACI}$ ante las NIIF; así mismo, prever la posición del movimiento cooperativo en su aplicación o rechazo.

\section{Referencias bibliográficas}

Agreda Moreno, J. E. (2012). El cooperativismo de trabajo asociado al derecho y al revés. Cali, Colombia: Facultad de Ciencias de la Administración, Universidad del Valle.

Alpa, O. (2014). Declaración Cooperativa de Cartagena 2014. Cartagena, Colombia: III Cumbre Cooperativa de las Américas, 2 al 7 de noviembre. Recuperado de: https:// www.aciamericas.coop/IMG/pdf/declaracioncooperativacartagena2014.pdf 
Anónimo. (2013). Aplicación de NIIF sector cooperativo. Contacto Cooperativo, 10. Recuperado de: http://www.confecoopnorte.com.co/periodico/Periodico_Ed_35.pdf

Barberini, I. (2008). Correspondencia de ICA al IASB. Recuperado de: www.aciamericas. coop/.../ICApositionontheFASBpreliminaryvie

Bastidas Vialcanet, R. y Amat Salas, O. (2014). Efectos económicos de la primera aplicación de las normas contables adaptadas a la NIC 32 en las cooperativas Revista de Contabilidad,17(2), 201-211. Recuperado de: https://www.sciencedirect.com/science/ article/pii/S1138489114000338

Beaubien, L. (2011). Co-operative accounting: Disclosing redemption contingencies for member shares. Journal of Co-operative Studies, 44(2), 38-44. Recuperado de: https:// thenews.coop/wp-content/uploads/s5-Beaubien-132.pdf

Bruque Cámara, S., Hernández Ortiz, M. J., Moyano Fuentes, J. y Vargas Sánchez, A. A. (2002). ¿Son más competitivas las sociedades cooperativas? Un análisis en el sector de la distribución farmacéutica. CIRIEC, 42, 131-157. Recuperado de: http://www. redalyc.org/articulo.oa?id $=17404206$

Confederación de Cooperativas de Colombia. Seminario internacional sobre impacto de las nuevas normas internacionales de contabilidad en las cooperativas. (2005). Confecoop, Argentina, Buenos Aires. Recuperado de: https://confecoop.coop/comunicoop/2005/ flash-confecomunica-no-408-reacciones-del-movimiento-cooperativolatinoamericano-impacto-de-las-nuevas-normas-internacionales-de-contabilidad-enlas-cooperativas/

Cooperativas de las Américas. (s.f.). Principios y valores cooperativos. Recuperado de: http:// www.aciamericas.coop/Principios-y-Valores-Cooperativos-4456

Cooperativas de las Américas. (2005). Primera reunión del Grupo de Normas Internacionales de Contabilidad de la ACI. Glasgow, Escocia, 20 de mayo. Recuperado de: http://www. aciamericas.coop/Primera-reunion-del-Grupo-de?tema $=18 \& \mathrm{nb}=90$

Cooperativas de las Américas. (2006). XIV Conferencia Regional competitividad y posicionamiento cooperativo: retos y oportunidades para el desarrollo económico y social, 17 al 21 de julio, Lima, Perú. Recuperado de: https://www.aciamericas.coop/XIV-Conferencia-Regionalde-ACI

Cooperativas de las Américas. (2012). Conclusiones del XII Congreso Nacional CooperativoColombia. Cartagena, Colombia. Recuperado de: http://www.aciamericas.coop/ Conclusiones-del-XII-Congreso

Cooperativas de las Américas. Región de la Alianza Cooperativa Internacional. (2014). Propuesta para la Coordinación Cooperativa Internacional respecto a las Normas Internacionales de Contabilidad. III Cumbre Cooperativa de las Américas. Cartagena, Colombia del 2 al 7 de noviembre de 2014. Recuperado de: https://www.aciamericas. coop/IMG/pdf/oscaralpa.pdf

Cooperativas de las Américas. (2015). Grupo de Trabajo NIC-ACl Américas. Recuperado de: https://www.aciamericas.coop/Grupo-de-Trabajo-NIC-ACI-Americas

Dussel, E. (2013). 16 tesis de economía política. Buenos Aires, Argentina, Editorial Docencia. Recuperado de: https://enriquedussel.com/txt/Textos_Obras_Selectas/(F)28.16_Tesis_ economia_politica.pdf 
Rincón, C., Molina, F. y Narváez, J. Perspectiva de la Alianza Cooperativa Internacional...

Fernández-Lorenzo, L. E. y Geba, N. E. (2005). Contabilidad social y gestión en ONGs. Revista Venezolana de Gerencia, 10(32), 545-563. Recuperado de: http://www.redalyc. org/articulo.oa?id=29003202

Gaitán Sánchez, Ó. M. (2014). Guía práctica de las entidades sin ánimo de lucro y del sector solidario. Bogotá, Colombia, Cámara de Comercio de Bogotá. Recuperado de: http:// bibliotecadigital.ccb.org.co/bitstream/handle/11520/8345/Guia Practica Entidades sin Animo de Lucro.pdf?sequence $=1$

Gómez, P. y Miranda, M. (2006). La caracterización financiera y contable del capital social a la luz de los principios cooperativos. Revesco, 90, 7-27. Recuperado de: https:// revistas.ucm.es/index.php/REVE/article/view/REVE0606330007A

Gómez, O., y Álvarez, R. (2013). Mediciones a valor razonable en la contabilidad financiera. Cuadernos de Contabilidad, 14(35), 441-461. Recuperado de: https://bit.ly/2LGQVg2

Green P., D. (2011). President of the Internation Year of Co-operatives Alliance. YouTube. Conference. Recuperado de: https:/www.youtube.com/watch?v=vxkWEn866N4

Hernández Rodríguez, D. R. y Escobar Castillo, A. E. (2017). Modelo de contabilidad social como herramienta de gestión para la responsabilidad social empresarial. IJMSOR, 2(1), 44-56. Recuperado de: http://webcache.googleusercontent.com/search?q=cac he:bJ800OaMPRMJ:ijmsoridi.com/index.php/ijmsor/article/download/86/76 +\&cd =1 $\& \mathrm{hl}=\mathrm{es} \& \mathrm{ct}=\mathrm{clnk} \& \mathrm{gl}=\mathrm{co}$

IASB. (2010). El marco conceptual para la información financiera. Londres, Reino Unido, IASB, 28 pp. Recuperado de: https://bit.ly/32Qs9PA

IFRS, F. (2015). Fundamentos de las conclusiones del proyecto de norma marco conceptual para la información financiera. Londres, Reino Unido, IASB, 97 pp. Recuperado de: https://incp. org.co/Site/2015/info/archivos/es_final-word_basis_ed_conceptual-framework.pdf

Ijiri, Y. (1975). Studies in accounting research. Sarasota, USA, American Accounting Association. Recuperado de: http://aaahq.org/Research/Calls-for-Submissions/Call-For-Papers-SAR

Lasserre, G. (2008). El hombre cooperativo. Bogotá, Colombia, Universidad Cooperativa de Colombia. 115 pp. Recuperado de: https://bit.ly/2YtqC2w

López Pérez, M. V. y Rodríguez Ariza, L. (2003). Un caso de interdisciplinariedad en teoría de la contabilidad: los cambios contables desde el enfoque cognitivo-linguiístico. Encuentros Multidisciplinarios, 15, 1-15. Retrieved from https://repositorio.uam.es/ bitstream/handle/10486/680283/EM_15_5.pdf?sequence $=1$

Mirabal-González, Y., Alfonso-Alemán, J. L. y Barrios-Hernández, Y. (2011). Sistema de contabilidad social para las empresas cooperativas de la provincia de Pinar del Río. Avances, 13(4), 1-10. Recuperado de: http://www.ciget.pinar.cu/Revista/No.2011-4/ articulo/Contabilidad_Social.pdf

Mirabal González, Y. (2014). Diseño del sistema de contabilidad social para las empresas cooperativas agropecuarias. Revista Cooperativismo y Desarrollo, 2(1), 1-16. Recuperado de: https://bit.ly/2YtqUq8

OCDE. (2016). Principios de Gobierno Corporativo de la OCDE y del G20. París, Francia, Editions OCDE. Recuperado de: http:/www.comex.go.cr/media/1899/principios-de-gobiernocorporativo-de-la-ocde-y-del-g20.pdf 
Palacios, F., Cardona, M. y Sánchez, M. del P. (2012). Relación empresas solidarias y organizaciones sociales productivas: análisis de caso. Tendencias y Retos, 17(2), 101115. Recuperado de: https://dialnet.unirioja.es/servlet/articulo?codigo $=4929258$

Pérez-Pascual,A.(2015). ¿Por quéfalla la economía?Economía Informa,393, 82-98. Recuperado de: https://www.sciencedirect.com/science/article/pii/S0185084915000298

Porter, M. E. (2002). Ventaja competitiva. Madrid, España: Alay Ediciones.

Rendón Álvarez, B., Montaño Orozco, E. y Gaitán León, G. (2013). Las NIIF y su impacto en las cooperativas en Colombia a diciembre 31 de 2011. Cuadernos de Administración, 26(50), 176-185. Recuperado de: http://www.scielo.org.co/pdf/cuadm/v29n50/ v29n50a07.pdf

Revista Integración Cooperativa. (2015). $\mathrm{N}^{\circ} 22$. Recuperado de: https://www.aciamericas. coop/Integracion-cooperativa-Numero-22

Rincón Soto, C. A. (2016). La contabilidad como un juego de lenguaje. Cuadernos de Administración, 32(55), 32-46. Recuperado de: http://www.scielo.org.co/scielo. php?pid=S0120-46452016000100004\&script $=$ sci_abstract\&tlng $=$ es

Robb, A. (2012). Stewardship: The core of cooperative accounting. Journal of Co-Operative Accounting and Reporting, 1(1), 19-27. Recuperado de: http://www.smu.ca/webfiles/A2JCAR-2102-1-1.pdf

Santander, P. (2011). Por qué y cómo hacer análisis del discurso. Cita Moebio, 41, 207-224. Recuperado de: http://webcache.googleusercontent.com/ search?q= cache:tQt3mbYA2QoJ:www.uchile.cl/documentos/santander_74716_0. $\mathrm{pdf}+\& \mathrm{~cd}=1 \& \mathrm{hl}=\mathrm{es} \& \mathrm{ct}=\mathrm{clnk} \& \mathrm{gl}=\mathrm{co}$

Suárez-Llanos, J. de G. y Manso Olivan, R. (1998). La contabilidad como lenguaje. Cuadernos de Administración, 24, 115-121. Recuperado de: http://bibliotecadigital.univalle.edu. co/bitstream/10893/2056/1/La contabilidad como lenguaje.pdf

Valentinov, V., Hielscher, S. y Pies, I. (2015). Nonprofit organizations, institutional economics, and systems thinking. Economic Systems, 39, 491-501. Recuperado de: https://www. sciencedirect.com/science/article/abs/pii/S0939362515000278?via\%3Dihub

Zubiaurre Artola, M. Á. (2004). Sociedades Cooperativas. Aspectos Contables Singulares. Cuadernos de Gestión, 4(2), 47-62. Recuperado de: http://www.ehu.eus/ cuadernosdegestion/documentos/424.pdf 